\title{
En direct de www.lamaisonducancer.com
}

\section{Rêvons un peu de traitements sans la fatigue!}

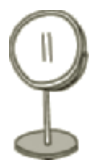

Oncomagazine a choisi d'ouvrir régulièrement ses colonnes à La-maison-du-cancer.com, site sociétal dédié à la manière de vivre le cancer au quotidien. Nous vous proposons de retrouver cette fois-ci le témoignage d'Anouchka, qui évoque la fatigue, cette " plainte $n^{\circ} 1$ des patients ».

$\mathrm{T}$ ous les cancérologues de ce monde se sont penchés sur nos maux, lors de la plus grande messe annuelle d'oncologie début juin (à l'American Society of Clinical Oncology, ASCO). Dans la recherche de thérapies de plus en plus innovantes semble poindre le désir de prendre en considération la qualité de vie des patients. Il est certain que ceux qui, comme vous et moi, pourront bientôt écrire un guide des effets secondaires, en seront bien aise. Car si nous approuvons ô combien tout ce qui concoure à notre "survie", nous ne serions pas fâchés de la troquer contre une vraie " vie ».

Imaginez que nos oncologues et chercheurs réussissent $L A$ prouesse, celle d'inventer des traitements qui n'induiraient plus cette terrible fatigue. Bien sûr, le cancer serait toujours là, avec son lot de médication, d'incursions à l'hôpital, de peur, de douleur, d'handicap, etc. II n'empêche, la fatigue, c'est la plainte numéro un des patients.

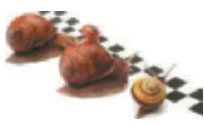
Pour ceux qui ne la connaissent pas de l'intérieur - à faire lire à vos proches !-, petite description du symptôme. Disons le tout de suite, pas grand-chose à voir avec la fatigue que l'on peut rencontrer en étant surmené de boulot. Rétrospectivement, je me contenterais bien de celle-ci ! Car finalement, quelques semaines de vacances, et hop, effacée. Non, cette fatigue-là relève du grand âge. Voilà un corps encore jeune, emmuré prématurément dans un corps de grand vieillard. II se réveille fatigué de la nuit ou de la sieste. Bref, le repos n'y change pas grand-chose. Fini le temps où il bondissait d'une pièce à l'autre, partait à grandes enjambées acheter le beurre oublié, envisageait plusieurs rendez vous dans une même journée, gérait mille choses en même temps, etc. Là, la vie ne s'envisage plus qu'au travers de priorités absolues. Concentrer le peu de son énergie sur un minimum de tâches ou de plaisir. Le reste ? Ça part à vau-l'eau. La maison pas rangée, les vêtements empilés, les coups de fil pas passés, les factures qui traînent, etc. Tout est effort, effort insurmontable souvent.
Alors imaginons que la fatigue ne fasse plus partie du programme de soins. À la condition d'être exempts de séquelles physiques handicapantes, nous pourrions continuer de travailler. Voire assumer d'avoir un cancer, puisque la maladie n'entraverait pas trop au bon fonctionnement de l'entreprise. Pour les salariés indépendants, leur activité ne coulerait pas puisqu'elle ne serait plus pénalisée par un entrepreneur shooté de fatigue. Non mais imaginez encore un peu. Nous ne dirions plus non aux invitations de notre entourage, évitant ainsi le repli sur nous ; nous réjouirions à l'idée de participer aux projets de notre conjoint et de nos enfants, bref, une vie sociale et affective rendue possible. Fini la culpabilité car nous reprendrions la main sur toutes ces tâches matérielles et ingrates déléguées faute d'énergie. En un mot, nous voilà maintenus dans la vie professionnelle, affective et sociale. Et puis une vie moins plombée par une fatigue abyssale et quotidienne, c'est déjà un risque moindre de dépression.

Bien sûr, cela ne résoudrait pas tout. Mais un gigantesque pas serait franchi vers une bien meilleure qualité de vie. Alors, faites vite, s'il vous plaît, car nous autres sommes pour I'heure bien à la peine. •

Anne-Laurence Fitère

www.lamaisonducancer.com est un site d'information sur le cancer à destination des malades, de leurs proches, du personnel soignant et de toute personne sensibilisée à ce fléau. Il cherche notamment à répondre aux difficultés professionnelles, administratives et financières, mais aussi relationnelles, esthétiques, pratiques qui se posent aux malades. Son objectif est ainsi de soulever le problème de la « double peine » qui les frappe. Le site entend également créer une communauté dynamique et active pour réussir à changer le regard de la société sur les malades du cancer. 